\title{
A concordância nominal de número interna ao DP: uma visão a partir do Programa Minimalista
}

\author{
Este trabalho é parte inicial de minha pesquisa \\ de mestrado. Quero destacar aqui meus \\ agradecimentos à FAPEAL pela bolsa de estudos \\ e às valiosas críticas da Prof ${ }^{a}$ Denilda Moura \\ durante apresentação na disciplina de Sintaxe. \\ Faz-se desnecessário ressaltar que qualquer \\ incoerência é de minha inteira responsabilidade.

\section{Jomson TeIXEIRA DA Silva Filho} \\ Mestrando do Programa de Pós-Graduação em \\ Letras e Linguística da Universidade Federal de \\ Alagoas.
}

Resumo: $O$ objetivo deste trabalho é uma breve descrição do processo de concordância nominal de número, abordando principalmente questões referentes à estrutura funcional do DP, às diferentes manifestações dos padrões de marcação morfológica de número nas línguas e sua relação com a interpretabilidade do traço de número, e também ao(s) mecanismo(s) de concordância sintática envolvido(s).

Palavras-chave: Agree; Determiner Frase; Concordância nominal de número; Programa Minimalista; Compartilhamento de Traços
Abstract: This paper is a brief description of the concord of number, especially addressing issues concerning the functional structure of DP, the various manifestations of patterns of morphological marking of number in language and its relation to the interpretability of the trace number, and also (s) mechanism (s) involved syntactic agreement(s).

Keywords: Agree; Determiner Frase; DP number agreement; Minimalist Program; Feature sharing 



\section{Introdução}

Tendo com objetivo a descrição da concordância nominal de número interna ao DP (sintagma determinante), passaremos em vista alguns trabalhos que analisam a estrutura do DP, desenvolvidos no quadro do Programa Minimalista (PM), bem como abordarei questões relativas à operação Agree, principalmente as propostas de Chomsky (1998; 1999) e Frampton \& Gutmann (F\&G), (2000). Estes apresentam uma novidade em relação à operação Agree segundo a qual há compartilhamento de traços, e não apenas checagem/valoração.

Conforme veremos, tal proposta parece mais interessante para o tratamento dos fenômenos de concordância no DP.

A organização do trabalho é a seguinte: no item 1 , passamos em vista o conceito da categoria lingüística de número e conceitos que darão base para as outras seções. No item 2, apresentamos alguns dados retirados da literatura que serão analisados de acordo com a proposta esboçada. Na seção 3 , apresentamos os principais

${ }^{1}$ Cf.: Ferrari Neto (2003) conceitos do Programam Minimalista (PM), seguido de uma breve descrição do processo de concordância no PM. Apresentamos por fim, as considerações finais.

\section{A Categoria Linguística de Número}

Pode-se definir número como uma categoria gramatical que leva em conta a quantidade de indivíduos designados nos nomes (CÂMARAJR., 1992). Essa categoria tem sido objeto de estudo de pesquisadores preocupados com sua manifestação lingüística em termos fonológicos, morfossintáticos e semânticos, em sua representação mental, em termos de acesso e representação lexical, nos problemas relativos à sua posição na estrutura do sistema computacional da língua como um traço formal ou como uma categoria funcional e em sua aquisição.

Essas pesquisas ${ }^{1}$ revelam um tema extremamente complexo e de grande dificuldade descritiva, devido à 
riqueza com que o número gramatical se manifesta nas diferentes línguas do mundo.

Um conceito que pode ser apresentado aqui como um dos mais freqüentes, apesar de haver outras acepções é a de "classe" ou "conjunto", referindo-se a qualquer grupo de elementos reconhecidos na descrição de línguas particulares (cf. LYONS, 1979). Essas "classes" ou "conjuntos" seriam traços universais da linguagem humana: cada língua manifestaria, em sua forma particular, categorias universais, como tempo, modo, caso, gênero, etc. $O$ número seria, assim, uma dessas categorias universais.

De fato, o caráter universal da categoria gramatical de número poderia ser atestado por estar presente em grande parte das línguas conhecidas no mundo. Uma observação minuciosa indica uma vasta e diferente manifestação dessa categoria. (CORBETT, 2001). É possível encontrar indicações da categoria de número na sintaxe de um nome, na morfologia, no léxico e na fonologia. A semântica do número também é muito variada, ocorrendo distinções entre valores de número muito além da famosa distinção entre singular e plural.

Em relação ao português brasileiro (PB) adulto, sabe-se que esse apresenta variação na marcação morfológica de plural (cf., p. ex., SCHERRE 1988; 1991; 1996):

(I) a. As camisas brancas (PB1 - marcação redundante)

b. As camisa branca (PB2 - marcação nãoredundante)

Da mesma forma, outros trabalhos (FERRARI NETO, 2003; LOPES, 2004, 2006; SIMIONI, 2007) demonstram que as crianças em fase de aquisição do $\mathrm{PB}$ "copiam" a variação encontrada nos dados de fala adulta.

Faz-se interessante ressaltar que os dados evidenciam uma grande variação não só entre crianças, 
mas também entre a fala adulta e a fala infantil, e mesmo na fala de uma mesma criança, conforme é possível comprovar nos exemplos que se seguem:

(II) Marcação não-redundante, apenas em D:
a. é as fruta (G., 2;3)
b. e os jacaré (A.C., 3;7)
c. os pauzinho (E., 2;9)

(III) Marcação redundante, em todos os elementos do DP:
a. ele quer lavar as mãos (A.C., 2;3)
b. agora vamos tirar os pregadores (G., 2;8)
c. as patas $(\mathrm{E} ., 3 ; 0)$

No entanto, além dos padrões exemplificados em (II) e (III), também encontramos no PB infantil dados como os de (IV):

(IV) Marcação agramatical, apenas em N:
a. a pessoas (E., 3;2)
b. aqui tem uma coisas (A.C., 2;8)

Sem dúvida alguma, essa variedade é agramatical no PB adulto, não estando, portanto, pelo menos em tese, presente no input a que a criança tem acesso.

Aqui serão analisados os dados do PB comparandoos com dados do italiano, do inglês retirados de corpora já constituídos na literatura. Também serão explorados dados do francês, sendo, todos estes dados também disponíveis em outros trabalhos que serão devidamente citados conforme se faça necessário.

\section{Apresentando alguns dados}

Correndo o risco de ser redundante, retomemos algumas proposições abordadas no item 1 . Analisando os dados do PB, do inglês e do italiano, podemos estabelecer 
algumas distinções bastante interessantes no que concerne aos padrões de marcação de concordância de número no DP, conforme é possível perceber pelos exemplos abaixo, retirados de Simioni (2007):

(1) As casas vermelhas são bonitas. (PB)

(2) As casa vermelha são bonita(s). (PB)

(3) As casa vermelha é bonita. (PB)

(4) The red houses are beautiful. (inglês)

(5) Le case rosse sono belle / La casa rossa è bella. (italiano)

Nos dados de (1) a (5) acima exemplificados, vemos que o inglês apresenta o determinante no singular, o nome no plural e o adjetivo no singular, destacando-se ainda o fato de que não há marcação de concordância de gênero. No italiano, por sua vez, a marca se manifesta em todos os elementos flexionáveis do sintagma. A marcação de gênero e número nessa língua é expressa pelo mesmo morfema (morfema cumulativo).

No caso do PB, temos dois padrões: em (1), o PB padrão, semelhante ao italiano, com marcação redundante de número, entretanto com morfemas independentes para gênero e número; e em (2), numa variedade mais coloquial, temos marcação de número apenas no determinante (e opcionalmente no adjetivo), como no inglês, mas diferenciando-se pelo fato de haver marcação na concordância de gênero.

O exemplo em (3) também pertence a um registro mais coloquial; temos aí que o verbo é e o adjetivo bonita (possivelmente predicado de uma Small Clause complemento do verbo ser, cujo sujeito é o DP) não concordam em número com o DP sujeito; entretanto, o adjetivo apresenta concordância de gênero com o nome casa.

Já os exemplos de (6) a (9) abaixo são aceitos por alguns falantes do PB e não aceitos por outros, o que torna esses dados duvidosos; É interessante observar a aparente opcionalidade na marcação de número. 
(6) ?As casa vermelhas são bonitas.

(7) ??As casa vermelhas são bonita.

(8) ??As casa vermelhas é bonita.

(9) ??As casa vermelhas é bonitas.

Os exemplos de (10) a (13) abaixo apresentam padrões que não são atestados na fala adulta nas línguas analisadas, o que os tornam agramaticais.

(10) ${ }^{*}$ A casas vermelha(s) [...]

(11) * Le casa rossa [...]

(12) * La case rossa [...]

(13) ${ }^{*}$ Thes reds houses $[\ldots]$

Com base nessas assunções, discutiremos propostas para a concordância e a estrutura do DP.

\section{O Programa Minimalista}

O Programa Minimalista é uma das versões mais recentes da Teoria Gerativa e não se apresenta como um novo aparato teórico, mas ao contrário, parte do modelo de Princípios e Parâmetros para propor uma série de orientações teóricas que objetivam especificar as condições que a faculdade da linguagem humana deve satisfazer para se desenvolver e determinar as propriedades que esta faculdade deve possuir.

O Programa Minimalista é orientado principalmente pela ideia de que devem ser evitados todos os postulados teóricos que são redundantes, ou seja, que não sejam estritamente necessários à teoria, derivando-se daí, o adjetivo minimalista.

De acordo com esse programa, as línguas humanas são formadas basicamente por um sistema computacional e um léxico. O léxico se apresenta como o lugar onde são representadas as propriedades individuais (particulares) 
${ }^{2}$ Confira Chomsky (1995) para um esclarecimento mais detalhado.

${ }^{3}$ Princípio de Interpretação Plena. de itens lexicais. Cabe ao léxico especificar os elementos que o sistema computacional seleciona para produzir expressões da língua. Dessa forma, um item lexical armazenado no léxico é composto por traços, por exemplo, $\mathrm{o}$ traço que indica a que categoria um item qualquer pertence (traço categorial - N, V, P, etc.) e os traços- $\varphi$ (traços de concordância - pessoa, gênero e número).

Propõe ainda dois níveis de representação que atuam como níveis de interface com os sistemas de desempenho. O nível de representação Phonetic Form (PF) se relaciona com o nível de interface articulatórioperceptual (A-P) e o nível Logic Form (LF) atua como interface com o sistema conceitual-intencional (C-I), não existindo mais níveis de estruturas lingüísticas para além dos níveis de interface PF e LF. Dizendo de outro modo não há mais Estrutura-D e estrutura-S. ${ }^{2}$ Para o momento em que a derivação separa os objetos de cada nível de representação, Chomsky postula o conceito de spell-out.

Uma língua particular L é concebida como um procedimento gerativo que constrói pares $(\pi ; \lambda)$. $\pi$ é a representação que corresponde à Forma Fonética (PF) e $\lambda$ a representação correspondente à Forma Lógica (LF). Os pares $(\pi ; \lambda)$ são interpretados nas interfaces A-P e C-I como comandos para sistemas de desempenho.Uma língua $\mathrm{L}$ especifica uma série de computações (derivações) que são responsáveis pela geração de expressões. De acordo com os postulados do Programa Minimalista, uma expressão de $L$ é pelo menos um par $(\pi ; \lambda)$ que satisfaça ao Full Interpretation $^{3}$ de acordo com o qual as interfaces PF e LF não possuem elementos que não podem ser interpretados por elas em seus respectivos sistemas de performance.

Vale ainda ressaltar que a cada derivação lingüística é feita, a partir do léxico, uma seleção de itens lexicais que são mapeados em uma expressão lingüística que disponibiliza informação para A-P e C-I. A derivação será convergente no nível da interface se for legível para esse nível, caso contrário, a expressão implodirá na interface. A computação é convergente se a expressão 
converge em todas as interfaces. O sistema computacional da linguagem humana seleciona dentre todas as expressões convergentes, aquelas formadas de modo mais econômico, ótimas, como por exemplo, sem passos redundantes na derivação ou apenas com movimentos locais.

Quanto aos traços, esses podem ser interpretáveis ou não-interpretáveis, intrínsecos ou opcionais. É interpretável quando pode ser lido nos níveis de interface, intrínseco quando seu valor já vem especificado na entrada lexical e é opcional quando seu valor pode variar, ou seja, é especificado quando é selecionado para a numeração. Assim, a especificação de caso e de traços- $\Phi$ é acrescentada a um item lexical no momento em que é selecionado para a numeração.

$\mathrm{Na}$ numeração, os traços- $\Phi$ e o caso são especificados ou pela entrada lexical (traços intrínsecos, gênero, por exemplo) e outros pela operação que forma a numeração (traços opcionais, de plural, por exemplo).

Concernente à interpretabilidade, não há ainda um "consenso" de onde o traço de número deve ser interpretável, se nos nomes ou em outros itens. Chomsky afirma que o traço de número é interpretável apenas no nome e não interpretável nos determinantes.

Ainda seguindo os passos do Programa Minimalista, categorias lexicais possuem traços [+ interpretável]. Por sua vez, as categorias funcionais possuem traços [- interpretável]. Entretanto já existem autores que contradizem a posição assumida por Chomsky (cf. MAGALHÃES, 2004).

\section{A concordância no programa minimalista}

O mecanismo de concordância proposto por Chomsky (1998) para o nível da sentença consiste na operação Agree, na qual uma sonda (Probe) com traços- $\Phi$ não interpretáveis $([\mathrm{u}])$ checa seus traços contra um alvo (Goal) com traços- $\Phi$ interpretáveis ([i]). Os traços- $\Phi$ são traços formais (pessoa, número e gênero) não- 
interpretáveis nas categorias funcionais, das quais devem ser eliminados antes do momento em que a derivação é enviada para os níveis de interface, ou seja, Spell-out.

A operação Agree permite, através da checagem de traços, o apagamento (erasure) dos traços não-interpretáveis da sonda e do traço não-interpretável de Caso do alvo, evitando que a derivação imploda (crash) em LF.

As condições para que Agree aconteça são as seguintes:

a sonda \pm deve possuir traços-\$ não-interpretáveis e c-comandar o alvo ${ }^{2}$ (o alvo deve estar no domínio de complemento da sonda); os traços de sonda e alvo tem que combinar (deve haver identidade); não deve haver um alvo alternativo $\gamma$ tal que $\alpha$ c-comande $\gamma$ e $\gamma$ c-comande $\beta$; e, finalmente, o alvo $\beta$ deve estar ativo para o sistema, isto é, deve possuir um traço não interpretável de Caso a ser checado. Ao ser inserida na derivação, a sonda \pm sonda seu domínio de complemento em busca de um elemento nominal ${ }^{2}$ ativo; ao encontrálo, \pm entra em relação de Agree $\operatorname{com} \beta$, checando e apagando seus traços não-interpretáveis; ao mesmo tempo, $\alpha$ checa e apaga o traço de Caso de $\beta$, que é não-interpretável e também deve ser eliminado antes de Spell-out.

Chomsky (1995 e obras posteriores) propõe a existência de dois mecanismos de concordância: Agree e Concord. Agree seria a concordância descrita acima, no nível da sentença, enquanto Concord funcionaria, via Merge, apenas no nível do sintagma.

Chomsky não entra em detalhes sobre o funcionamento de Concord, mas, segundo Simioni (2007), parece claro que ele não toma este mecanismo como uma operação sintática strictu senso, e sim como um mecanismo morfológico de concordância. Além disso, Chomsky assume que os traços- $\Phi$ são interpretáveis em $\mathrm{N}$ (como dito anteriormente), dessa forma, a concordância no $\mathrm{DP}$ aparece sobre modificadores e $\mathrm{D}$, mas é $\mathrm{N}$ que rege essa concordância. 
Chomsky (1999) propõe uma alteração na operação Agree. Agora a interpretabilidade dos traços é determinada já no léxico, com distinção de valores: traços nãointerpretáveis entram na derivação sem valor especificado, enquanto os traços interpretáveis entram com valores especificados. A operação Agree valora e apaga os traços não-interpretáveis da sonda e o Caso do alvo.

A proposta de Frampton \& Gutmann (2000a) para a operação Agree mantém algumas características da proposta de Chomsky conforme apresentada acima, ao mesmo tempo em que traz reestruturações interessantes.

F\&G (2000a) apresentam um conceito de concordância sintática no qual dois nós terminais distintos incluem um mesmo traço. De acordo com essa perspectiva, Agree implica compartilhamento de traços; traços interpretáveis ou não-interpretáveis que combinam (matching features) se juntam em um único traço compartilhado, que é valorado se um dos traços combinados tiver valor. Essa idéia substitui aquela em que um traço valorado dá seu valor a uma contraparte não valorada.

Outro detalhe que diferencia da proposta original é que traços $[\mathrm{u}]$ se juntam tanto quanto um traço $[\mathrm{u}] \mathrm{e}$ um [i]; a operação Agree é induzida por traços [u] e é cega aos valores dos traços.

F\&G (2000a) afirmam haver dois tipos de traços relevantes para o estabelecimento da concordância sintática: traços-б e traços-\$; elementos $\mathrm{N}$ vêm do léxico com seus traços- 1 e traços- $\$$ valorados, enquanto os atribuidores de Caso vêm sem valor. Afirmam ainda, não haver indicação direta de Caso na sintaxe; Caso é atribuído após a sintaxe, com base no compartilhamento de traços: nominais recebem marcação de Caso porque compartilham um traço- $\Phi$ com um atribuidor de Caso. O requerimento de que um nominal seja marcado para Caso é, portanto, morfológico.

Segundo este modelo uma derivação ocorre da seguinte maneira: a operação Select introduz os núcleos 
${ }^{4}$ Para mais de talhes cf. Simioni (2007), ou ainda a obra original dos autores F\&G (2000). A obra está em inglês.

${ }^{5}$ Cf.: Name (2002). (pivot) na sintaxe; logo após, a operação Attract satisfaz os traços [u] do pivot conduzindo uma busca top-down e uma busca externa para o caso de a primeira não funcionar; as operações Select e Attract de cada pivot constituem um ciclo. O efeito de interveniência é traduzido nos seguintes termos: a busca top-down não continua após o encontro de um matching feature, mesmo que este seja $[\mathrm{u}] .^{4}$

Os autores propõem ainda a existência de núcleosTE (TE-heads), que desempenhariam um papel na atribuição de Caso e na visibilidade dos argumentos para o componente interpretativo. Outra noção fundamental para essa proposta é a noção de cadeia.

Frampton \& Gutmann (2000a) formulam uma condição sobre cadeias que diz que cada cadeia- $б$ deve ser encabeçada por um núcleo-TE e cada núcleo-TE deve ser a cabeça de uma cadeia-1.

\section{A concordância e a estrutura do DP}

Além da concordância que se estabelece entre sujeito e verbo e objeto e verbo, há também relações de concordância que se estabelecem entre determinantes, modificadores e nome dentro do DP e que segundo Chomsky (onforme já observado), é controlada pelos traços do nome.

Ferrari Neto (2003), afirma que os primeiros trabalhos a estudarem a concordância nominal, ou seja, aquela que se manifesta entre os elementos do DP, preocuparam-se basicamente na explicação da realização de gênero e número no nome e não exatamente em fazer uma descrição da concordância entre nome e determinante. ${ }^{5}$ Diante disso, pode-se afirmar que a descrição da concordância nominal feita nos primeiros momentos seguiu os mesmos passos que nortearam a descrição da concordância verbal supracitada.

Sendo assim, a concordância nominal passou também a ser entendida como o resultado de operações de movimento de núcleos lexicais para núcleos funcionais, 
onde receberiam a flexão correspondente aos traços de gênero e numero.

A estrutura do DP assumida por estes trabalhos pressupunha a existência de núcleossintáticos responsáveis pela atribuição de valores de traços de gênero e numero dentro do DP. Estes núcleos seriam projeções dos traços de gênero e número, constituindo assim as categorias funcionais GenP e NumP.

O que se pode observar do exposto acima é que essas concepções acabam por marcar uma diferenciação entre os momentos anteriores da teoria gerativa e os que seriam propostos a partir daí, considerando em especial aspectos relacionados à caracterização do número gramatical, pois esse passa a ser visto como uma categoria funcional resultante de uma projeção sintática e não apenas como um traço formal.

Seguindo a linha histórica de Ferrari Neto (2003), pode-se afirmar que um dos primeiros trabalhos a seguir a linha acima delineada, foi o de Bernstein (1991). Esse autor utilizando dados do francês e do valão, estudou basicamente a manifestação de concordância entre adjetivos e nomes, propondo um movimento de NP para núcleos funcionais presentes dentro do DP para dar conta da marcação de número. ${ }^{7}$

Outra proposta com base na concordância no DP foi apresentada por Picallo (1991) na qual a concordância consistiria em inserir o nome na derivação sem marcas de gênero e número para depois elevá-lo até GenP $\mathrm{P}^{8}$. Lodo após haveria outro movimento do nome para NumP recebendo conseqüentemente o número. O que aparece de novo nessa proposta é o tratamento dado ao gênero, concebido como uma categoria funcional, diferente de outras propostas.

Diversamente da concepção de Picallo (1991), Ritter (1993), afirma que somente o número possui status de categoria funcional, NumP, sendo atribuído ao Nome no curso da derivação, com o movimento do nome para esta projeção. O gênero, contudo, continua sendo tratado como um traço formal, sendo atribuído ao nome em um 
dos núcleos do NP. Esse estudo se baseou em dados do hebraico, comparados as línguas românicas.

De acordo com Name (2002), a idéia de Picallo (1991) só se realizaria satisfatoriamente se aplicada aos nomes com traço de gênero opcional, fracassando no caso dos nomes com traço de gênero intrínseco, uma vez que sua concepção faz supor que um nome como mesa deveria ser alçado para GenP para receber gênero, mesmo sendo este um traço já especificado na entrada lexical de mesa.

$\mathrm{Na}$ Teoria de Checagem proposta (Chomsky, 1995), as relações de checagem ocorrem entre núcleo e especificadores. Para checar seus traços [- interpretável], um elemento de uma categoria funcional atrai o núcleo de uma categoria lexical no seu domínio. O núcleo se move para a posição Spec da categoria funcional, checando seus traços [+ interpretável] com os traços da outra categoria, que são posteriormente apagados.

Uma forma diferente desta operação de checagem é a que acontece entre Determinante e Nome, ou seja, entre núcleo e complemento. Chomsky a chama de Concord (já citado anteriormente) para separar a concordância com movimento (Agree), da concordância que envolve somente concatenação (merge), com checagem de traços in situ, ou seja, sem alçamento do Nome para uma posição de especificador.

Carstens (2000) afirma que esta operação de checagem entre Determinante e Nome aconteceria diferentemente. Ela assume um nível intermediário entre $\mathrm{NPe} \mathrm{DP}$, o NumP, que seria uma projeção do número (Num) na sintaxe. A categoria lexical D teria traços interpretáveis de pessoa e não-interpretáveis de gênero e número; Num, por sua vez, teria traço interpretável de número e $\mathrm{N}$ teria traço interpretável de gênero. $O$ traço não-interpretável de gênero de $\mathrm{D}$ motivaria o alçamento de $\mathrm{N}$, que seria alçado primeiramente para NumP, onde receberia número, e, em seguida, para $\mathrm{D}$. $\mathrm{N}$ seria adjungido a $\mathrm{D}$.

Vale ressaltar mais uma vez que proposta

de Carstens fornece interessantes modificações das 
formulações iniciais de Chomsky (1995). A mais notável delas é aquilo que foi anteriormente citado de forma indireta, a saber, a criação de uma categoria funcional para o número, o qual, segundo esta concepção, sairia do léxico deixando de ser tratado como um traço formal presente na entrada lexical do nome para ser incluído na sintaxe como uma projeção.

Observemos abaixo a estrutura do DP proposta por Carstens (2000,p. 328)

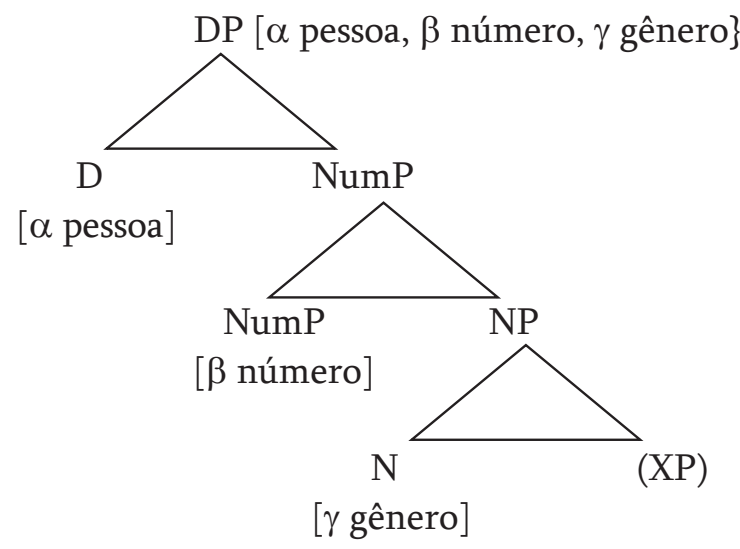

Magalhães (2004) propõe um mesmo mecanismo de concordância para os níveis da sentença e do DP, a saber, a operação Agree. A autora retoma o trabalho de Chomsky (1999) sobre a valoração de traços em construções participiais e propõe que "a valoração dos traços formais do DP se dá da seguinte maneira: primeiro, os traços $\varphi$ dos concordantes são valorados entre eles e em seguida, o DP tem seu caso valorado por uma sonda" (MAGALHÃES, 2004, p. 156).

Além disso, Magalhães apresenta uma novidade ao discutir em que núcleo do DP o traço de número seria interpretável. Baseada em Abney (1987), Olsen (1989) e Longobardi (1994), a autora afirma que há evidências de que o traço de número, pelo menos no que tange ao $\mathrm{PB}$, seja interpretável nos determinantes, contrariamente à hipótese de Chomsky, segundo a qual, 
conforme já dito anteriormente, o traço de número é interpretável nos nomes.

A partir da proposta do DP apresentada por Abney (1987), Magalhães (2004) hipotetiza que D tenha traço interpretável de número e não-interpretável de gênero. Todos os outros constituintes do sintagma teriam traços não-interpretáveis.

A autora ainda afirma que mesmo em línguas em que o morfema de número apareça apenas no nome como é o caso do inglês, há evidências de que o traço seja interpretável no determinante.

É importante ressaltar que esta autora não assume a projeção Num como o núcleo de uma categoria funcional independente, conforme podemos perceber a partir da estrutura a seguir

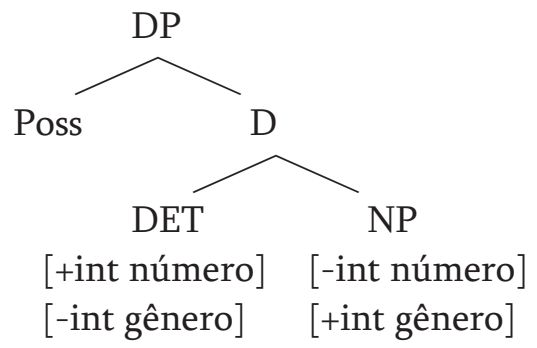

Com essa proposta, Magalhães (2004) explicando os fenômenos de concordância no PB afirma para Abney (1987) há uma relação de s-seleção entre D e N semelhante à existente em IP entre sujeito e verbo:

No DP, D é o núcleo que seleciona o NP como complemento do mesmo modo que o VP funciona como complemento para Infl. Temos assim uma relação argumento/predicado entre $\mathrm{D}$ e $\mathrm{N}$ no sintagma nominal e sujeito e verbo no sintagma verbal. Na sentença, os traços de número são interpretáveis no sujeito (argumento) e nãointerpretáveis no verbo (predicado). O mesmo acontece no DP: traços de número são interpretáveis no $\mathrm{D}$ (argumento de $\mathrm{N}$ ) e não-interpretáveis em $\mathrm{N}$ (predicado). (MAGALHÃES, 2004, p. 161) 
A seguir, apresentamos um exemplo de derivação retirado Magalhães (2004, p. 159):

(15) As casas vermelhas

(a) vermelhas casas $[-\mathrm{n} /-\mathrm{g} /-\mathrm{K}] \quad[-\mathrm{n} /+\mathrm{g} /+\mathrm{p} /-\mathrm{K}]$

(b) vermelhas casas $[-\mathrm{n} /+\mathrm{g} /-\mathrm{K}] \quad[-\mathrm{n} /+\mathrm{g} /+\mathrm{p} /-\mathrm{K}]$

(c) $\mathrm{D}$ vermelhas casas $[+n /-g /-K] \quad[-n /+g /-K] \quad[-n /+g /+p /-K]$

(d) $\mathrm{D}$ vermelhas casas $[+\mathrm{n} /-\mathrm{g} /-\mathrm{K}][+\mathrm{n} /+\mathrm{g} /-\mathrm{K}][-\mathrm{n} /+\mathrm{g} /+\mathrm{p} /-\mathrm{K}]$

(e) $\mathrm{D}$ vermelhas casas $[+n /-g /-K][+n /+g /-K][-n /+g /+p /-K]$

(f) $\mathrm{D}$ vermelhas casas $[+\mathrm{n} /+\mathrm{g} /-\mathrm{K}][+\mathrm{n} /+\mathrm{g} /-\mathrm{K}][+\mathrm{n} /+\mathrm{g} /+\mathrm{p} /-\mathrm{K}]$

(g) $\mathrm{T} \quad \mathrm{D}$ vermelhas casas $[-n /-p][+n /+g /-K][+n /+g /-K][+n /+g /+p /-K]$

(h $\mathrm{T} \quad \mathrm{D}$ vermelhas casas $[+\mathrm{n} /-\mathrm{p}][+\mathrm{n} /+\mathrm{g} / \mathrm{NOM}][+\mathrm{n} /+\mathrm{g} /-\mathrm{K}][+\mathrm{n} /+\mathrm{g} /+\mathrm{p} /-\mathrm{K}]$

(i) $\mathrm{T} \quad \mathrm{D}$ vermelhas casas $[+n /-p][+n /+g / N O M][+n /+g /-K][+n /+g /+p /-K]$

(j) $\mathrm{T} \quad \mathrm{D}$ vermelhas casas $[+\mathrm{n} /-\mathrm{p}][+\mathrm{n} /+\mathrm{g} / \mathrm{NOM}][+\mathrm{n} /+\mathrm{g} / \mathrm{NOM}][+\mathrm{n} /+\mathrm{g} /+\mathrm{p} /-\mathrm{K}]$

(k) $\mathrm{T} \quad \mathrm{D} \quad$ vermelhas casas $[+\mathrm{n} /-\mathrm{p}][+\mathrm{n} /+\mathrm{g} / \mathrm{NOM}][+\mathrm{n} /+\mathrm{g} / \mathrm{NOM}][+\mathrm{n} /+\mathrm{g} /+\mathrm{p} /-\mathrm{K}]$

(l) $\mathrm{T} \quad \mathrm{D}$ vermelhas casas $[+\mathrm{n} /+\mathrm{p}][+\mathrm{n} /+\mathrm{g} / \mathrm{NOM}][+\mathrm{n} /+\mathrm{g} / \mathrm{NOM}][+\mathrm{n} /+\mathrm{g} /+\mathrm{p} / \mathrm{NOM}]$ 
Analisando a estrutura acima, podemos perceber que em (a), o nome casas entra na derivação com seus traços de número e Caso não valorados, enquanto o adjetivo vermelhas entra na derivação com todos os seus traços não valorados.

Em (b), vermelhas sonda e entra em relação de Agree com casas, valorando seu traço de gênero. Em (c), vemos que $D$ entra na derivação, sonda e entra em relação de Agree com vermelhas, valorando o traço de número do último, mas não o traço de gênero de $\mathrm{D}$; isso faz com que D sonde casas (passo (e)), valorando seu traço de gênero e o traço de número do alvo. Por fim, uma sonda $\mathrm{T}$ entra na derivação e sonda cada um dos elementos do DP, valorando os traços de Caso dos mesmos. Ao sondar D (g), a sonda T valora seu traço de número e ao sondar casas $(\mathrm{k})$, seu traço de pessoa.T

A análise de Magalhães (2004) parece dar conta dos dados, mas não está livre de problemas. Simioni (2007) afirma que a ordem linear do adjetivo não corresponde à ordem observada no $\mathrm{PB}$ e ainda o fato de a marca morfológica de número ser expressa em $\mathrm{N}$ no inglês parece representar um problema, pois Magalhães (2004) associa a interpretabilidade do traço ao núcleo em que a marca morfológica é realizada.

\section{5. À guisa de uma conclusão}

Um dos argumentos para se adotar a hipótese de Magalhães, é que pelo menos ao que diz respeito ao $\mathrm{PB}$, com enfraquecimento da concordância a marca morfologicamente visível de número seria mantida apenas no núcleo em que esse traço seria interpretável, assim o morfema optaria por permanecer na posição em que estaria situado o determinante como se observa em exemplos de fala como "nós vai" ou "as menina".

Além do mais, a hipótese de Magalhães (2004) parece dar conta satisfatoriamente do mecanismo de concordância interna ao DP sem a necessidade de postular um mecanismo a mais, "concord". 
Pensarmos ainda nos dados do $\mathrm{PB}$, em que algumas variantes apresentam marca morfológica de número aparece apenas sobre o determinante (como no exemplo "as meninas") é possível imaginarmos que é $\mathrm{D}$, por ser a cabeça da cadeia- $\sigma$, assumindo assim, como fazemos nesse trabalho a ideia de Agree como apresentada em F\&G (2000a), o responsável pela definitude e pela interpretação semântica em LF, carregando a marca de plural. Nos casos em que as variedades do PB apresentam marca morfológica em todos os elementos do sintagma, como os dados do italiano, podemos considerar que a marca se alastra por conta do compartilhamento de traços.

Quanto ao fato de o inglês não apresentar marca visível de concordância em $\mathrm{D}$, uma possível solução para esse impasse seria a suposição de que em línguas como o inglês haveria um morfema zero de plural no determinante, levando em conta o caso dos determinantes demonstrativos these, those em que há marca de plural manifestada morfologicamente. Ou ainda, seguindo a linha de raciocínio de Simioni (2007), pode-se pensar que a língua não forneça os meios para que o plural seja expresso em $\mathrm{D}$ (exceto no caso dos demonstrativos), e isso faz com que a marca apareça sobre outro elemento que compartilha o traço de número com a categoria em que esse traço seria interpretável, em D.

Diante dos argumentos acima enumerados, assumimos neste trabalho a hipótese dessa autora para dar conta dos dados analisados nesse trabalho. Faz-se necessário ressaltar, no entanto, que uma pesquisa que apresente mais dados e mais detalhes o que vem sendo feito no trabalho de mestrado de Silva Filho (2011), em andamento. Esperamos assim esboçar uma proposta que dê conta dos fatos observados nas línguas naturais. 


\section{Referências}

ABNEY, Steven Paul. The English noun phrase in its sentential aspect. Tese (Doutorado em Lingüística) Massachusetts Institute of Technology (MIT), Cambridge, 1987.

CARSTENS, Vicki. Concord in minimalist theory. Linguistic Inquiry, Cambridge, v.31, n.2, p.319-355, abr. 2000.

CHOMSKY, Noam. The minimalist program. Cambridge: MIT, 1995.

Minimalist inquirires: the framework. In: MIT Occasional Papers in Linguistics. Cambridge, n.15, 1998.

Derivation by phase. In: MIT Occasional Papers in Linguistics. Cambridge, n. 18, 1999.

FERRARI NETO, José. Reconhecimento do número gramatical e processamento da concordância de número no sintagma determinante na aquisição do Português Brasileiro. Tese (Mestrado em Lingüística), - PUCRJ, 2003.

FRAMPTON, John. \& GUTMANN, Sam. Agreement Is Feature Sharing. ms, 2000a.

LONGOBARDI, Giuseppe. Reference and Proper Names: a Theory of Movement in Syntax and LF. Linguistic Inquiry, Cambridge, v.25, p.609-665, 1994.

LOPES, Ruth. Estágios no processo de aquisição de número no DP do Português Brasileiro. Letras de Hoje, Porto Alegre, v.39, n.3, p.157-171, 2004.

MAGALHÃES, Telma. M. V. A valoração de traços de concordância dentro do DP. DELTA, São Paulo, v.20, n.1, p.149-170, 2004. 
NAME, M. C. L. Habilidade Perceptuais e Lingüísticas na Aquisição e Processamento da Concordância de Gênero. Tese de Doutorado. PUC/RJ: Departamento de Letras, 2002

OLSEN, Susan. AGR(eement) in the German Noun Phrase. In: BHAYY, LÖBEL, SCHMIDT (eds.). Syntactic Phrase Structure Phenomena in Noun Phrase \& Sentences. Amsterdam: John Benjamins, 1989, p.39-49.

RITTER, Elizabeth. Two functional categories in noun phrases: evidence from modern Hebrew. In: ROTHSTEIN, Susan (ed). Syntax and Semantics 25: Perspectives on Phrase Structure. New York: Academic Press, 1991.

SIMIONI, Leonor. Aquisição de concordância nominal de número: um estudo de caso. Trabalho de conclusão de curso, UFRGS, 2004.

A concordância de número no DP: propostas minimalistas. Estudos Lingüísticos XXXVI(1), janeiro-abril, 2007. p. 117 / 125.

A aquisição da concordância nominal de número no português brasileiro: um parâmetro para a concordância nominal. (Mestrado em Linguística), - UFSC, 2007. 